\title{
Practical Guidance on How to Handle Levodopa/Carbidopa Intestinal Gel Therapy of Advanced PD in a Movement Disorder Clinic
}

\author{
Stephen Wørlich Pedersen*, Jesper Clausen and Mie Manon Gregerslund
}

Movement Disorder Clinic, Department of Neurology, Copenhagen University Hospital Glostrup, Denmark

\begin{abstract}
Continuous dopaminergic delivery is recognized for the capacity to ameliorate symptoms in Parkinson's disease (PD). In advanced PD the short comings of orally administered Levodopa/Carbidopa include fluctuations resulting in unstable effect and dyskinesia. Levodopa/Carbidopa intestinal gel, LCIG, (Duodopa®, Abbott Laboratories) is delivered continuously through a percutaneous endoscopic gastrostomy with the inner tube placed in the duodenum by means of a device (CADD legacy Duodopa pump (CE 0473)). The therapy implies continuous dopaminergic delivery directly to the duodenum and is therefore unaffected by gastric emptying and represents a major adjuvant in the treatment of advanced PD with significant improvement in motor and non-motor symptoms. The aim of this paper is to suggest the prerequisites for a LCIG clinic and propose a feasible set-up and lean organization of a movement disorder clinic. Secondly, the paper proposes practical handling of patients in LCIG treatment for advanced PD based on experience and initiation of LCIG treatment and follow-up in forty patients.
\end{abstract}

Keywords: Administration, advanced PD, continuous dopaminergic delivery, Duodopa, dyskinesia, fluctuations, LCIG, organization.

\section{INTRODUCTION}

The movement disorder Parkinson's disease (PD) is the second most common neurodegenerative disease. The prevalence of PD is $0.3 \%$ in industrialized countries and PD occurs in about $1 \%$ to $2 \%$ of populations $>60$ years of age. [13]. Pathologically, PD is characterized by the progressive degeneration and loss of dopaminergic neurons. Clinically,PD includes symptoms of rigidity, bradykinesia, resting tremor, gait disturbance or postural instability and non-motor symptoms of autonomic dysfunction, pain and sensory disturbances, sleep impairment, fatigue in addition to psychiatric symptoms. Replacement therapy with dopaminergic drugs is required for symptom control [4]. Advances in drug development are essential for advances in treatment.

Levodopa has a short plasma half-life of approximately 90 minutes (when administered with Carbidopa), and is rapidly metabolized.In advanced $\mathrm{PD}$, most dopamine terminals have degenerated, leading to an unregulated and discontinuous dopaminergic delivery, which is believed to cause motor fluctuations and dyskinesias despite chronic therapy [5].

In advanced PD subcutaneous apomorphine, deep brain stimulation (DBS), or Levodopa/ Carbidopa intestinal gel (LCIG) (Duodopa ${ }^{\circledR}$, Abbott) are the treatments of choice [6]. LCIG has demonstrated significant improvement of motor symptoms in advanced PD by reducing motor fluctuations and dyskinesias [7-10]. Furthermore LCIG has demonstrated beneficial effect in a range of common PD non motor

*Address correspondence to this author at the Movement Disorder Department of Neurology Copenhagen University Hospital Glostrup, DK2600 Glostrup, Denmark; Tel: +45 43233468; Fax: +45 38633926;

E-mail: stephen.w.pedersen@ regionh.dk symptoms such as cardiovascular symptoms, sleep disturbance, attention/memory deficits, gastrointestinal, and urinary symptoms as well as pain [8-10].

The literature lacks descriptions of how to set up and handle LCIG and how to avoid errors. This paper aims to describe the practical circumstances required to handle LCIG treatment at a movement disorder clinic. The set-up, procedures, systems, and checklists are described with the purpose of sharing the key learnings and practical experiences from treating more than 40 patients with LCIG over a period of four years in a movement disorder (MD) clinic specifically designed to initiate and follow-up patients eligible for LCIG treatment.

\section{PART I: BASIC REQUIREMENTS TO A MOVEMENT DISORDER CLINIC FOR IMPLEMENTING LCIG THERAPY}

The authors of this paper have documented the processes and systems used during patient work up and initiation of therapy, and defined roles and responsibilities. This paper is a retrospective review of the key learnings from setting up an LCIG clinic in addition to a prospective summary of essential requirements that were found necessary along the way. On an organizational note, a flat matrix organization at the MD clinic, allows for a smooth, non-hierarchical, easy going and lean workflow. Routines are characterized by mutual professional respect with empowerment of the individual staff member in his or her patient handling to the benefit of the patients.

The Movement Disorder Clinic at Copenhagen University Hospital Glostrup handles LCIG and apomorphine therapy, while patients eligible for DBS are referred to other centers. Two movement disorder specialists, a senior physician, 
and a specialized PD nurse are working full time at the MD clinic seeing about 1000 patients a year. The majority of these patients $(85-90 \%)$ have PD or atypical PD. LCIG requires a PEG (percutaneous endoscopic gastrostomy) through which the drug is administered continuously by an external device [6, 11-13].

A movement disorder clinic that is specialized in PD needs endorsement and back up financially and practically from the hospital and departmental management. Commitment and motivation to implement radical changes are required from all health care professionals involved in the clinic as well as in the remainder of the neurology department. In order to set up the LCIG clinic, training and education of staff are required prior to introducing the therapy as a treatment modality.This implies internal training of neurology department staff, radiologists, gastroenterologists, and emergency room staff in addition to external training of patients, relatives, primary caregivers and health care professionals. Wide spread education in basic as well as in advanced PD, handling of the pump and Levodopa/ Carbidopa titration are required. Additionally, skills training or continuous training may also be offered by the manufacturer.

In the process of establishing an LCIG clinic, reference material needs to be produced and communicated to the interns and fellows on call at the neurology department in order to provide education on how to handle questions and issues that may come up outside the opening hours of the LCIG clinic.

The start-up of an LCIG clinic is a long term commitment, requiring sufficient patient flow in order to maintain skills and gain sufficient practical experience, secure routines and observational standards for admission and discharge. An LCIG clinic should preferably have at least twothree movement disorder specialists and an MD specialist nurse with special expertise in PD. Furthermore procedures must be well established and systematically followed. At the MD clinic at Glostrup Hospital 4-6 patients are started in LCIG treatment a year. Finally, it is sensible prior to setting up the LCIG clinic to consider the structure of local or national reimbursement and reflect on whether this treatment modality is financially feasible for PD patients attending the clinic.

\section{Accessibility to Gastroenterology and Radiology Departments}

Patients attending the clinic need to have access to the gastroenterology and the radiology department, during the initiation phase of LCIG for establishment of PEG as well as during follow-up, where tubal problems may need check-ups with X-ray. In case of tubal misplacement a rapid intervention from the gastroenterologists are instrumental to continued treatment. The roles and responsibilities of the gastroenterologists must be clearly defined and dedicating just a couple of gastroenterologists will allow them to familiarize and get the expertise of handling the special tubes used for LCIG. A close collaboration between a few committed gastroenterologists and the staff at the MD clinic will facilitate the patient handling and flow. This is also important for the subsequent collaboration on stoma care. The routines on referrals and rapid handling are based on the mutual under- standing between the different departments, that these patients cannot wait days or weeks before being examined. Sub-acute handling is often required to avoid unnecessary hospitalizations due to technical issues disrupting the treatment temporarily. Additionally, trouble shooting in cases of emergency complications after discharge need to be in place, ensuring that the treatment concept is well known to all the departments.

\section{Establishment of an Internal and External Emergency Function}

The MD clinic at Glostrup Hospital has established an emergency function in order to provide assistance for patients around the clock and through holidays and weekends. The emergency function is handled by the neurology department and by the out-patient clinic. Outside office hours the emergency function is handled by a third party healthcare service call center. At the call center medically qualified staff takes the calls from the patient and troubleshoot on the pump and PEG issues, and they are trained specifically to handle LCIG and PEG issues. The third party call center is funded by the manufacturer of Duodopa, Abbott and is open from 06:00 to 24:00. Albeit, the emergency function is provided by a third party, the responsibility of patient care remains that of the MD clinic at all times. The emergency function must be established before the start-up of the first patient. This implies having an internal backup system during office hours and that patients know that in case of problems they can always contact the outpatient clinic or the neurology ward for help.

\section{External Collaborators}

There may be countries or regions where private practicing neurologists work community based and where LCIG treatment is not an option, or where different treatment conditions and routines apply. However, in cases of decentralized patient handling by private practicing neurologists, patients may be referred to larger neurology centers where LCIG is offered. If community based neurologists are used for handling advanced PD patients locally, the referral system to MD clinics for LCIG eligible patients needs to be in place and they must be informed on the opportunities of this treatment modality for their patients.

The MD clinic at Glostrup Hospital takes referrals from all over the country, but currently LCIG is offered at six hospitals in Denmark. At present these centers count about 104 patients on LCIG in total. In order to share the knowledge and experiences as well as providing sufficient material to do quality assurance, it seems relevant to establish a national LCIG / PD database to collect relevant data on this treatment modality.

The professional support and engagement from the national Neurologists' Society and Society of Movement Disorder Specialists are instrumental for successful implementation of LCIG treatment. The Danish Patient Association on PD has embraced LCIG as a new treatment modality and internally the association has established an LCIG patient network for patients to share the experiences on LCIG. 


\section{LCIG, a Medicinal Product and a Device}

LCIG therapy requires a high degree of internal and external collaboration. The set up implicates close collaboration internally at the neurology department, with gastric surgeons, the radiology department, and externally with home caregivers, as well as relatives of the PD patient.

LCIG is a gel developed for continuous intestinal administration. The gel should be administered directly into the duodenum or upper jejunum by a permanent tube via percutaneous endoscopic gastrostomy (PEG) and with a portable external pump (CADD legacy Duodopa pump (CE 0473)). A positive test of the clinical response to LCIG administered via a temporary nasoduodenal tube is required before a PEG is inserted [14].

The dose should be adjusted to an optimal clinical response reflecting individual needs of each patient. This is for the purpose of maximizing the functional $\mathrm{ON}$-time by minimizing the amount of OFF time.

\section{PART II: GETTING STARTED}

\section{Pre-treatment Phase- in the LCIG Outpatient Clinic}

\section{Patient Selection}

LCIG is indicated for treatment of advanced levodoparesponsive Parkinson's disease with severe motor fluctuations and hyper-/dyskinesia when available combinations of Parkinson medicinal products have not given satisfactory results [14].

Long and/or frequent OFF periods and/or severe dyskinesias in spite of optimized oral therapy are situations where LCIG may be considered, and when treatment of advanced symptoms by means of DBS or continuous subcutaneous infusion with apomorphine is contraindicated, ineffective, or otherwise unsuitable. The patient selection is based on the clinical assessment made by a neurologist specialized in movement disorders. The definition of a therapy resistant, yet Levodopa-responsive patient is dependent on a previous good response to Levodopa and is difficult to quantify. A partial good response, i.e. general clinical improvement on the UPDRS to oral Levodopa has been suggested as guidance [10]. However, the clinical impression of a treatment effect remains greatly based on individual experience with the treating physician.

LCIG may be used in the elderly people. Dementia is not per se a contraindication for LCIG in cases where relatives can handle the therapy and the device. The outlook for patients with dementia becoming less physically dependent as the motor deficits improve is positive. Still, LCIG should be avoided in patients with severe dementia. Absolute contraindications for the use of LCIG comprise: hypersensitivity to Levodopa, Carbidopa or any of the excipients, narrow-angle glaucoma, severe liver and renal insufficiency, severe heart failure, severe cardiac arrhythmia, acute stroke, non-selective MAO inhibitors and selective MAO type A inhibitors must not be given concomitantly, and should be withdrawn at least two weeks before initiation of LCIG treatment, conditions in which adrenergics are contraindicated, e.g. pheochromocytoma, hyperthyroidism and Cushing's syndrome [14]. Finally, limited access to caregivers or physical distance between the patient's home and the LCIG clinic may also be inhibiting factors for initiation of the LCIG treatment.

Patients are usually started in pairs of two in order for them to support each other during the test process. The in depth knowledge to the different patients allow the nurse and physicians to assess the individual patient's capacities and needs, and match the patients to start LCIG treatment in pairs of two.

\section{Patient Information and Expectations}

Information to patients should be given both orally and in writing. Before obtaining informed consent patients should be allowed sufficient time to fully consider the implications of LCIG treatment. If the patient consents to have the trial test performed, further in-depth information concerning the therapy is discussed with the patient. Meeting patient expectations is very important for the establishment of a successful LCIG treatment result. Patient and relatives need to understand what the realistic and expected results are and what potential complications may occur during treatment. Patient and relatives need also to comprehend that the treatment is expected to be life-long or as long as benefit is maintained. It is advisable also to document this information being given to the patient. Discussing thoroughly with the patient what is realistic to obtain is essential in this phase to avoid post discharge disappointment and complaints based on unrealistic patient expectations. All of these discussions and necessary reflections must be dealt with and completed prior to the trial test.The care giver burden before LCIG and after must be taken into consideration and discussed with the patient and relatives.

\section{Patient Evaluation}

Rating scales may be used to evaluate the patient prior to the treatment and after the initiation and may comprise MiniMental State Examination (MMSE), Hoehn and Yahr, Bartel score, Unified PD Rating Scale (UPDRS), and non-motor symptom scale. The evaluation of the function level, Activity of Daily Living (ADL), physical ability and evaluation of quality of sleep may also be assessed on admission by a physiotherapist, occupational therapist, and by the general ward staff. These observations are added in order to evaluate all the general effects of the treatment that are unlikely to be captured by classical rating scales used in PD.

\section{Treatment Initiation Phase- in the Ward}

\section{LCIG Initiation}

A step-by-step procedure to be strictly followed during admission to assess the effects of LCIG with a nasal trial tube and the circumstances around this, as well as the procedures if treatment is continued by inserting a PEG tube are listed in detail in APPENDIX A.

During the test phase the patient is admitted to the neurology department, preferably attended by the LCIG clinic staff during day time.The admission routines around testing and titration are characterized by verbal repetition for reassurance to the patient on what is going to happen. Many PD patients experience anxiety to the outlook of medicine discontinuation, but the familiar setting and reassurance from staff that the patients know well has proven beneficial. Day 1 
is a day of preparation. At the end of the first day, discontinuation of standard PD medication must take place and will inevitably make the patient hypokinetic or akinetic the following morning. Patients must be informed to expect worsening - and if necessary temporary use of sedatives for the night and potentially anxiolytics may be considered. Day 2 is the actual first day of LCIG testing and days 3-5 are used for titration.

\section{Dosing and Titration}

The total daily dose of LCIG is calculated from the total dose of oral Levodopa prior to initiation of LCIG therapy, and the morning dose of LCIG is calculated from the morning dose of oral Levodopa. The continuous daily dose of LCIG is the calculated total daily dose of LCIG minus the calculated morning dose, divided by 16 (i.e. therapy given continuously over 16 waking hours). In order to reduce hyperkinesias the morning dose of LCIG is reduced slightly (80\%) compared to oral Levodopa, The total daily oral dose is converted 1:1 to continuous daily dose of LCIG. Thus morning doses range from $2.5-7.0 \mathrm{ml}$ and maintenance doses range from $2.4-5.0 \mathrm{ml}$. The pre-programmed settings cannot be erased by the patient. Table $\mathbf{1}$ displays an example of how to calculate the LCIG dose from the dose of oral Levodopa.

Administration of an extra bolus may be given as required if the patient becomes hypokinetic during the day and the patient is instructed on how to administer a bolus dose. The extra dose is adjusted individually; normally $0.5-2.0 \mathrm{ml}$. Higher doses may be needed. If the need for extra bolus doses exceeds 5 times per day the maintenance dose should be increased. Dose adjustments are based on clinical impression; however a suggested and empiric titration schedule is outlined in Table 2.

In order to simplify the calculation of LCIG dose, it may be considered to switch patients on PD polytherapy to monotherapy oral Levodopa. After switching to monotherapy deterioration can be expected after two-three days, thus it is recommended to allow a least a week for potential dose adjustments prior to admission and LCIG initiation.

\section{Establishment of PEG}

Depending on the clinical response, it is decided during day 3-5 whether to continue the treatment and whether the PEG may be inserted during the same admission. Scheduling for PEG insertion needs to be accounted for upfront when planning the admission. If the PEG is to be established postdischarge, the patient may continue on LCIG after discharge with the nasal tube and revert shortly for a PEG. PEG establishment is described in APPENDIX B. Patients may wish to make their decision on PEG establishment and continued LCIG treatment after being at home in their normal surroundings. During this period of testing the treatment and the device and reflecting on the implications, a nasal tube is used for administration of medication.

\section{Preparation to Discharge}

When the titration level is reached and either the PEG has been established or planned for in the near future, the patient is prepared for discharge. This involves thorough instruction and information to patient and caregiver on how to handle

Table 1. Conversion of Oral Levodopa to LCIG Equivalent

\begin{tabular}{|l|l|l|l|}
\hline \multirow{2}{*}{} & \multirow{2}{*}{ Oral Levodopa Dose } & \multicolumn{2}{|c|}{ LCIG (Duodopa ${ }^{\circledR}$ 20 mg/ ml) } \\
\cline { 3 - 4 } & & Calculated Morning Dose 80\% of Oral Dose & Calculated Continuous Dose 100\% of Oral Dose \\
\hline Morning dose & $100 \mathrm{mg}$ & $100 \mathrm{mg} / 20 \mathrm{mg} / \mathrm{ml}=5 \mathrm{ml}$ & \\
\hline Total daily dose & $1000 \mathrm{mg}$ & & $\begin{array}{l}1000 \mathrm{mg}-100 \mathrm{mg}=900 \mathrm{mg} \\
900 \mathrm{mg} / 20 \mathrm{mg} / \mathrm{ml}=45 \mathrm{ml} \\
45 \mathrm{ml} \text { Duodopa } / 16 \mathrm{hours}=2.8 \mathrm{ml} / \mathrm{hour}\end{array}$ \\
\hline LCIG Start- up dose & & & $2.8 \mathrm{ml} /$ hour $=\mathbf{2 . 8} \mathbf{~ m l} / \mathbf{h o u r}$ \\
\hline
\end{tabular}

Example of how to calculate the LCIG morning and continuous dose from the oral Levodopa morning dose and total daily dose, respectively. In the example the patient receives 100 $\mathrm{mg}$ Levodopa as a morning dose and $1000 \mathrm{mg}$ as the total daily oral dose. LCIG (Duopdopa ${ }^{\circledR}$ ) is $20 \mathrm{mg} / \mathrm{ml}$ ). The LCIG morning dose is set at $80 \%$ of the oral Levodopa dose, and the continuous dose is set at $100 \%$ the total daily dose minus the morning dose.

Table 2. Suggestion for LCIG Titration based on Clinical Efficacy and Adverse Reactions

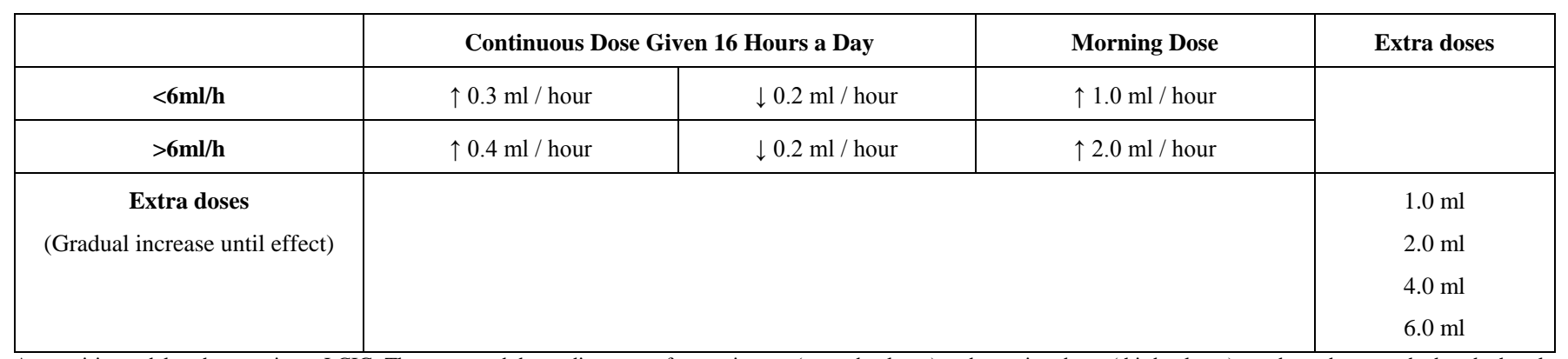

An empiric model on how to titrate LCIG. The suggested dose adjustments for continuous (second column) and morning doses (third column) are dependent on whether the hourly dosing is above or below $6 \mathrm{ml} / \mathrm{h}$ (first column). Increases are made due to clinical impression of inadequate response and decreases due to hyperkinesias or other adverse reactions. Extra doses are given - also based on clinical impression, $1 \mathrm{ml}$ at the time. The patient can administer extra doses, however these should not exceed 5 times a day as this implies the need for an adjustment of the continuous daily dose. 
the pump, trouble shooting in case of technical problems, and caretaking of the PEG.

Instructions on the pump are provided by the manufacturer, however familiarizing the patient with the device is obviously important, and this includes reassurance that the pump is pre-programmed and set on the maintenance dose and morning dose that has been medically justified during the titration phase. Morning doses and maintenance dosing are set individually. Fig. (1) displays patient flow and decision chart for test and titration of LCIG in advanced PD.

\section{Treatment Follow-up Phase-in the LCIG Outpatient Clinic}

\section{Patient Follow-up}

Clinical controls are scheduled regularly when the effect of LCIG treatment has stabilized. Control every 6 months may be feasible. It is important to allow for individual needs, some will find half year checkups appropriate while others are keen on returning for checkups more often. The easy accessibility to the clinic is reassuring and improves patients' self or caregiver's confidence in their capacity of handling

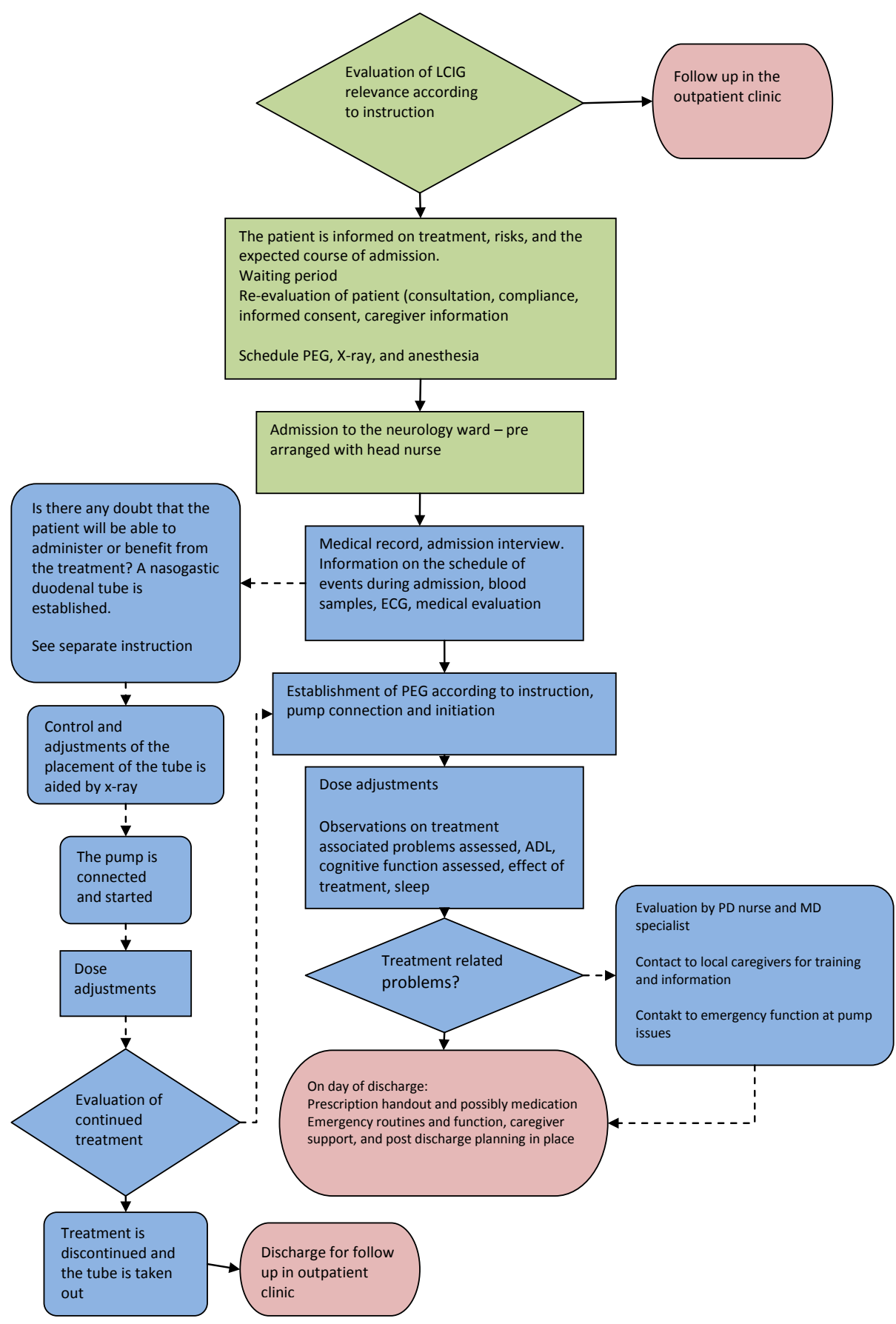

Fig. (1). Patient flow and decision chart for test and titration of LCIG in advanced PD. Green indicates procedures and instructions to follow at the out-patient clinic setting, blue - at the in-patient setting during the admission period for trial test, and red indicates discharge to followup at the out-patient clinic. 
the device and the treatment. Once a year the clinical controls are expanded to comprise already performed ratings in order to evaluate and assess possible changes in the effect, dose requirements, cognitive, and functional capacity. Furthermore the pump and PEG will get maintenance and service checkups once a year - or more frequently in cases of malfunction.

Continuous dopaminergic delivery with LCIG is effective in reducing both motor and non-motor deficits in advanced $\mathrm{PD}$, and specifically sleep disturbances and urinary problems seem to improve [7-10, 15]. Psychiatric disorders may have been overlooked due to the severe motor deficits, and after initiation LCIG treatment they may become apparent, uncovered when the hypokinesia and rigidity is no longer dominating. Definite psychiatric morbidity at pretreatment evaluation tends not to improve after LCIG treatment initiation. In fact it appears that psychiatric peculiarities possibly tend to flourish as the patient regains mobility. These issues need to be taken into account prior to treatment initiation and may result in discontinuation later.

\section{Patient Discontinuation}

Patients who do not respond clinically to LCIG during the test period will remain on oral therapy. In cases where patients initially respond during the test phase, but on a midterm or long term demonstrate little or no effect of LCIG the therapy should be discontinued. The issue of potentially being a non-responder must be discussed with patients and relatives upfront before starting LCIG and the establishment of a PEG. Patients who are discontinued from LCIG are subsequently transferred to oral PD therapy. However, the long term perspectives for the patients appear to be positive as data has demonstrated a positive long term effect of LCIG [16]. All together the aspects of watching advanced PD patients with severe motor and non-motor deficits improve sometimes radically are professionally encouraging and highly motivating.

\section{Patient Support}

Thorough information to the patients and relatives will minimize the risk of complications. Check lists and written instructions minimize errors or complications caused by the staff. Education familiarizes everyone at the neurology department getting involved with LCIG. This improves the level of trouble shooting, which reduces hospitalizations due to complications or malfunction of e.g. the pump or the PEG. A PEG issue, e.g. displacement, does not necessarily require hospital admission outside office hours of the outpatient clinic. The pump may easily be turned off while the patient takes pre-prescribed oral rescue medication, i.e. Levodapa / Carbidopa, and after contact to the movement disorder clinic the following morning, presents at the radiology department for a check up on the positioning of the tube.

The support groups provide a social network for people who share experiences, and in addition leave room for the exchange of knowledge between patients and relatives. These and other elements may be discussed and shared in larger patient support groups which have proven useful.

As the LCIG patients recover mobility new opportunities arise - and some patients may wish to travel. For that purpose cooling bags can be provided for carrying the medication along with an additional pump as back up. Information on how to handle this is provided by the manufacturer.

As for the primary care the patient's GP, homecare, nursing, and the local pharmacy are important collaborators, with whom the LCIG clinic needs to be in contact and work closely with about patient care. The PD nurse establishes the contact and provides the relevant information for these external collaborators in order to ensure optimal external patient support.

\section{Patient Cases}

\section{Case \# 1}

62 year-old woman with a long history of PD that progressively developed into unpredictable ON/OFF episodes of varying duration and severity. Despite optimization of the oral medication the functional level was greatly dissatisfactory. The patient received, limited by side effects, a maximum acceptable oral dose regime of dopamine agonist $8 \mathrm{mg}$ Ropiniol ER once a day, a total Levodopa medication with
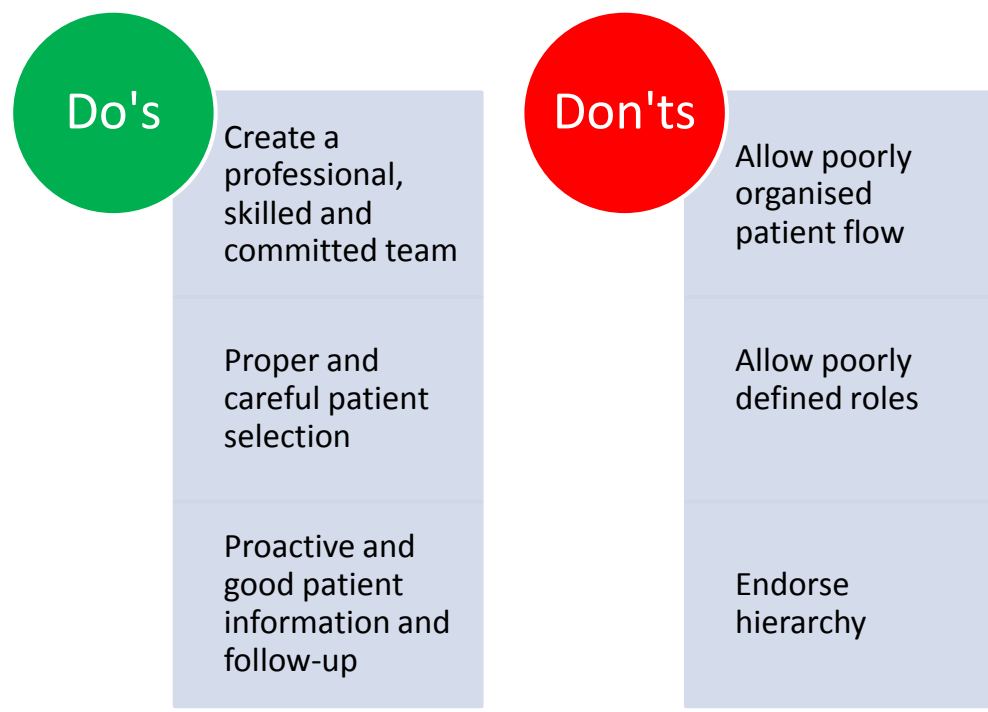

Fig. (2). A brief overview summarizing what to focus on - and what to avoid when starting an LCIG clinic 
$650 \mathrm{mg}$ Levodopa dispensed differently 6 times daily together with Entacapone. Doses were dispensed in an individually adjusted pattern. Compliance was optimized with no improvement in functionality. The patient was found eligible for LCIG therapy and was admitted to the hospital for LCIG test. After an uncomplicated insertion of nasoduodenal tube, discontinuation of all oral PD medication was done. LCIG therapy was initiated with increasing doses of Levodopa/ Carbidopa. The effect of LCIG was good and subsequently a PEG was inserted without complications during the admission. The patient was discharged for follow-up and titration of LCIG at the outpatient clinic. A couple of weeks after the initiation of LCIG the patient had achieved a stable functional level with only minor - acceptable - fluctuations during the day characterized by brief episodes of hyperkinesias. Stable dosages of LCIG were adjusted to a level where the morning dose was calculated to $8.5 \mathrm{ml}$, the continuous dose to $2.4 \mathrm{ml} / \mathrm{h}$ and extra doses of $1.5 \mathrm{ml}$ given two to three times daily.

During follow-up dose increases have not been required and despite some progression in symptoms her functional level remains unchanged with a high level of independence and an insignificant need for external care. After three years the patient is still in LCIG therapy with stable and acceptable sustained benefit receiving a morning dose of $6.5 \mathrm{ml}$, a continuous dose of $2.6 \mathrm{ml}$ and extra doses $1.5 \mathrm{ml}$ given two to three times a day.

\section{Case \# 2}

78-year old man with a long history of PD who over the past few years experienced an increasing number of fluctua- tions occurring during the day with unexpected ON/OFF episodes and hyperkinesias. Concomitantly with an increasing number of medically resistant fluctuations the patient developed progressive dementia with symptoms of Alzheimer's disease. The oral medication comprised orally optimal doses with dopamine agonist and Levodopa tablets with a total load of Levodopa of at least $1500 \mathrm{mg}$ daily dispensed up to 7 times during daytime. Levodopa was also dispensed at nights. The medication was well looked after by a well-functioning spouse. Despite the dementia the patient was well functioning at home given the care of his wife, but he experienced increasing difficulties with motor fluctuations resulting in severe motor impairment and an increase in dependency of the spouse. The patient was tested for LCIG, and after discontinuation of oral medication and insertion of a nasoduodenal tube the LCIG was initiated and adjusted over the following days. The clinical evaluation of the patient was that the overall effect of LCIG treatment appeared to be only moderate. However the general wellbeing of the patient seemed to be greatly improved. The patient was discharged from the hospital with a nasoduodenal tube and during the following six weeks the LCIG treatment was adjusted leading to a significant improvement in motor behavior. Treatment level with LCIG was a morning dose of $7.5 \mathrm{ml}$, a continuous dose of $4.0 \mathrm{ml} / \mathrm{h}$ and extra doses was $2.0 \mathrm{ml}$ given two times daily. In addition rescue medication with Levodopa tablets was allowed at nights. The cognitive function had also improved during this period. Albeit the dementia was evaluated as being in principle unchanged the motor function had been improved. The patient had a PEG inserted and continued LCIG with good effect. Despite being

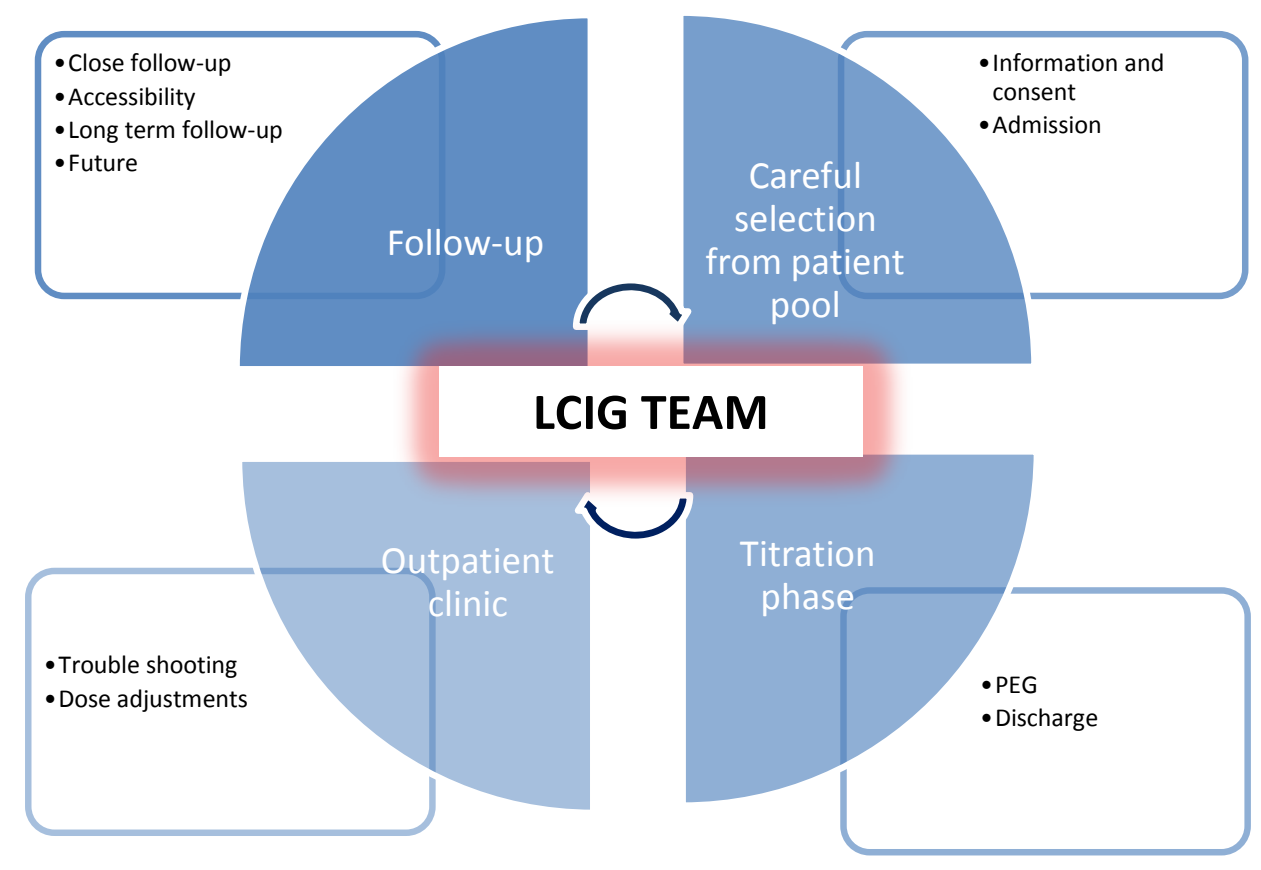

Fig. (3). An overview of the organization and essential elements of handling LCIG. The central element is a team of competent movement disorder specialists, physicians and nurses. After careful patient selection, information and consent, patients are admitted to the ward. During the titration phase the effect is evaluated and PEG is performed. After about five days the patient is discharged. Trouble shooting and subsequent dose adjustments are handled by the LCIG team in the outpatient clinic where the follow-up takes place. Easy accessibility to the clinic is essential allowing a close and frequent follow-up post discharge - and will gradually change and become more infrequent over time during long term follow-up. 
moderate to severely demented, the patient is still functioning independently to some degree with acceptable motor function.

\section{Case \# 3}

79-year old woman with progressive PD symptoms through 15 yearsleading to major impairment in functional level and problems in handling daily activities. The patient slowly became more and more dependent on a wheel chair for transportation over shorter and longer distances and was also dependent on professional home care being unable to manage on her own. In addition, she had cognitive impairment. The patient's PD symptoms were dominated by unpredictable OFF episodes of varying duration and severity. She received Levodopa/Carbidopa five times daily in combination with a monoamine oxidase $\mathrm{B}$ inhibitor once a day and Amantadine twice daily. Despite optimal compliance, the patient still had an unsatisfactory and impaired functional level. The patient was tested for LCIG, to see whether she could benefit from this treatment although it was a prerequisite for her to receive continuous care and assistance for handling of medication and device. Despite these considerations it was still a possibility that the patient would gain from the treatment. The patient was submitted to the hospital and after discontinuation of oral PD medication the LCIG was initiated after insertion of a nasoduodenal tube. The patient had a considerably longer titration period than usually anticipated, and the LCIG level was adjusted to a higher level compared to the oral dose of Levodopa/Carbidopa than prior to admission for LCIG test. During the test period it was noted that the patient had major cognitive impairment that were more outspoken than noticed previously prior to the test of LCIG. The LCIG treatment was well tolerated and without major side effects. As the patient appeared not to have any beneficial effect on motor capacity of the treatment, the patient was regarded as a non-responder and the LCIG treatment was discontinued. The patient returned to oral PD medication and during follow up her functional level remained unchanged and at the same level she had before admission for LCIG testing. Over the following years she further progressed with more Parkinson's symptoms and suffered from extensive functional loss of motor skills and increasing need for external help and daily care, until she died 2.5 years later.

\section{DISCUSSION}

The aspects of starting a lifelong treatment are issues of both ethical and practical substance. The clinical data as well as the clinical experience with the LCIG substantiates that a continuous dopaminergic delivery minimizes fluctuations in advanced PD. Administration directly to the duodenum via a PEG allows for absorption of Levodopa that is unaffected by gastric emptying. Although the drug is administered via a pump the device yields plenty of flexibility for individual dosing and adjustments to fit the requirements and medication needs of each patient. The LCIG treatment affects both the motor and the non-motor symptoms. In some cases the non-motor symptoms may be improved in-directly via the improvement of motor symptoms. Although, LCIG treatment does not have the capacity to change the progressive nature of the disease, it remains a major adjuvant to treatment in advanced PD.
It requires organized commitment from the MD clinic as well as from the hospital to initiate and continue LCIG treatment, because the consequences of discontinuation of this as a treatment option are radical to the patients. For discussions on the financial aspects around LCIG it should be taken into consideration what the economic burden it is to society. On the other hand the efficacy of LCIG is indisputable and will provide benefit for patients and relatives and ease the caregiver burden substantially. Figs. (2) displays main focus areas and what to aviod - and Fig. (3) displays in overview organizational and essential elements for successful implementation of LCIG in an outpatient movement disorder clinic.

\section{CONCLUSION}

This paper aims to describethe practical issues related to the start-up and maintenance of an LCIG outpatient clinic, leaving the reader with guidance and tools that may readily be implemented into other movement disorder clinics or adapted according to local standards and prerequisites. In the experience of the authors' trouble shooting flowcharts, written instructions and procedures, checklists, and follow-up improve patient care in advanced PD during LCIG treatment initiation and follow-up. Finally, the well-organized and well-functioning team work is regarded as instrumental for the successful implementation of LCIG. The practical guidance has the purpose of serving as inspiration to other clinics that may benefit from putting their own routines into system, simplifying clinical processes, and increase knowledge sharing among health care professionals.

\section{ABBREVIATIONS

$\begin{array}{ll}\text { ADL } & =\text { Activity of daily living } \\ \text { DBS } & =\text { Deep brain stimulation } \\ \text { LCIG } & =\text { Levodopa/Carbidopa intestinal gel } \\ \text { MD } & =\text { Movement disorder } \\ \text { MMSE } & =\text { Mini-mental state examination } \\ \text { PD } & =\text { Parkinson's disease } \\ \text { PEG } & =\text { Percutaneous endoscopic gastrostomy } \\ \text { QoL } & =\text { Quality of life } \\ \text { UPDRS } & =\text { Unified Parkinson's disease rating scale }\end{array}$

\section{CONFLICT OF INTEREST}

SWP has served on advisory boards for Solvay Pharma, Abbott, UCB, GSK and Desitin and has served as a consultant for Orion Pharma, and Ipsen.

$$
\begin{aligned}
& \text { JC: None } \\
& \text { MMG: None }
\end{aligned}
$$

\section{ACKNOWLEDGEMENTS}

The authors thank Charlotte Strøm, MD, PhD, for writing assistance with this paper. This service was financially supported by Abbott. Abbott had the opportunity to review and comment on the publication content; however, all decisions regarding content were made by the authors. 
APPENDIX A: Day by day description of the admission procedure for LCIG test

APPENDIX B: Procedures and description of the insertion of the duodenal tube for LCIG therapy

APPENDIX C: Procedures and instructions on post-operative observation and care of patients with Parkinson's disease following insertion of a PEG tube under local anesthetics for LCIG

\section{APPENDIX A: ADMISSION PROCEDURE FOR LCIG TEST}

\section{Method}

\section{Admission Day}

The patient attends the ward at 10.00 a.m.

- Admission consultation with nursing staff on the ward to check that the details on the patient registration chart are up-to-date. Contact person/doctor is appointed.

- Standard admission procedures in the unit are followed.

- The Parkinson's nurse/appointed staff provide information on the planned procedure and insertion of the trial tube.

- A nasal tube is inserted by nursing staff according to current guidelines. See special guideline.

- Once the tube is inserted, the patient is given advice on using the pump.

The Parkinson's nurse books an X-ray of the tube for the next morning, if this has not been done previously.

- The doctor prepares the admission records, orders blood tests and draws up the medication regimen. Specifically taken into account are: Previously prescribed medication, Sinemet/Madopar at night, Motillium. The study charts are filled in, if this has not been done, MMSE, Hoehn and Yahr, Bartel score, UPDRS and non-motor symptom scale. Check that an X-ray of the nasal tube has been booked for the following day.

- All Parkinson's medication is withdrawn at 00.00 hrs.

- The doctor calculates the Duodopa dose so it is ready when treatment can begin.

NB! The patient does not have to go without food or drink before the X-ray the following day.

Day 2

- Blood samples are taken at 8.00 a.m. on rounds as routine tests.

- The tube's position is determined by X-ray examination.

- When the tube is positioned correctly in the duodenum, the Duodopa treatment begins.

The rest of the day and evening is used to observe the effect of and adjust the therapy using neurological assessment. When the Parkinson's nurse goes home, the nursing staff takes over observation duties and clinical assessments.

The nasal tube remains in place for approx. 3-5 days before a decision is taken on whether actual therapy is continued or whether treatment should be stopped.

Days 3-5

- Daily adjustments of therapy and clinical assessment of the effect of Duodopa treatment is to be made. At the end of the period a decision on whether the patient is to undergo PEG insertion is taken.

If Therapy is to be Continued and PEG is to be Inserted During the Same Admission:

The patient must be informed about the PEG procedure by the attending doctor and must give his/her acceptance for insertion of a PEG tube and is noted in the medical records as well as an indication for insertion and the ASA score.

On the day prior to PEG insertion, relevant blood samples are taken - INR/Thrombocytes/BAS test, blood type, if this has not been done. The patient must fast for 6 hours before the surgical procedure and is not allowed to drink anything for 4 hours prior to the procedure. An intravenous drip is applied.

- The patient goes to the endoscopic unit for insertion of a PEG tube, taking with them:

- Medical records

- PEG tube and inner tube (Freka PEG set Gastric FR 15 / Freka Intestinal tube FR 9 for PEG 15 FR)

- Pump

The pump is switched off when the patient goes to the surgical unit, thus the pump is switched off approx. 30 minutes before inserting the PEG). The nasal tube remains and is removed by the gastroenterologist in the endoscopy unit.

- The PEG tube and inner tube is inserted. When the tube is inserted, therapy continues immediately.

- The nasal tube is extracted.

The patient may drink 2 hours after the procedure and eat soft/liquid food 4 hours after the surgical procedure and for the rest of the day so as to minimize abdominal discomfort after the insertion of the PEG tube. Normal diet can be resumed the next morning. 
Nursing care and observation of the tube given according to current guidelines. See guideline.

Discharging the Patient from the Ward with Duodopa Pump:

- Patient, relatives or home care giver must be advised on:

Pump functions, troubleshooting, relevant contact tel. no. (link to discharge with tube).

The patient is issued with the remaining medication, a Duodopa and Sinemet slow

release prescription and a certificate for use when travelling.

- The patient is given the additional equipment to take home.

Responsibility and Distribution of Tasks

- Leaders of the ward are responsible for seeing that the staff is familiar with the guidelines and that the physical framework and necessary aids are in place so that the guidelines can be followed.

- Individual members of staff are responsible for knowing and following the guidelines.

\section{APPENDIX B: DUODENAL TUBE FOR LCIG THERAPY}

\section{Method}

\section{Prescription by a Doctor:}

Indication: It is noted in medical records that there is a need to test the effect of Duodopa ${ }^{\circledR}$ before deciding to insert a PEG tube for continuous therapy.

Motilium 20mg tablets is prescribed, 3 tablets TID.

$\mathrm{X}$-ray examination of the inner tube position in the duodenum.

- Information

- Patient is given information about the procedures during the admission and the daily examinations.

- Before the nasogastric tube is inserted, the patient is informed of the indication for treatment and informed consent is obtained.

- Before and during insertion, the patient is told:

- that it is important to cooperate during placement of the tube

- that it can help to breathe steadily, slowly and deeply

○ to bend the head forward and drink water (a glass with a drinking straw) whilst the tube is passed down the oesophagus

- about discomfort in the form of a gag reflex when the tube passes the throat and back of the tongue

$\circ \quad$ that he/she is continuously guided and supported while the procedure is being carried out

Hygiene

The nurse and any assistants must wash and disinfect hands and use non-sterile disposable gloves during the procedure.

- Materials

- Tube, IPN-STABILIFE Duodopa®Naso-Intestinal Tube

- Drapes with plastic underside

- Tray and cellulose

- Installagel® lubricating gel

- Denture box, if applicable

- Glass of water possibly with drinking straw

- Fixation adhesive plaster

- $20 \mathrm{ml}$ syringe

- Non-sterile gloves

- Stethoscope

- Preparation and procedure for insertion of nasogastric tube for treatment with Duodopa ${ }^{\circledR}$.

1. The guide wire is fully inserted into the tube. This is attached to the connector.

2. The length of the section of the tube to be inserted via the nostrils is calculated as the distance of $85 \mathrm{~cm}$ (the distance from the wing of the nose to the stomach) added an additional $25 \mathrm{~cm}$ (the distance from the Pylorus to the Treits ligament in the Duodenum. The distance of $110 \mathrm{~cm}$ is marked on the tube.

3. The patient is placed in a comfortable sitting or semi-recumbent position.

4. The drape is placed under the patient's chin.

5. If the patient is wearing full or partial dentures, these are removed.

6. The tip of the tube is coated in Installagel ${ }^{\circledR}$

7. An assistant holds the tray with cellulose under the patient's chin and a glass of water and, if necessary, a drinking straw.

8. The tube is passed through the nose. When the tube reaches the throat, the patient's head must be bent forward, and the patient must make as many swallowing movements as possible. The tube is pushed down without pressing. It continues being inserted until the first marking is reached. 
9. The position of the tube is verified by aspiration or injecting air into the tube whilst listening for bubbling sounds above the epigastrium using a stethoscope.

10. When the position of the tube has been checked, at least $20 \mathrm{ml}$ of water is injected as a secondary control.

11. Approximately $25 \mathrm{~cm}$ of guide wire is withdrawn from the tube. Insertion then continues until the $110 \mathrm{~cm}$ marking on the tube. The guide wire can then be entirely withdrawn from the tube. NB: The guide wire is after removal cleaned and placed in a small plastic pouch marked with a label indicating the patient's name and personal identification number. The wire may be used if the placement of the tube needs to be adjusted.

12. The tube is attached to the nose and cheek with an adhesive plaster.

NB: If the patient coughs and becomes cyanotic the tube is withdrawn and a new attempt is made to insert the nasogastric tube after the patient is recovered.

Within 8-12 hours, gastric motility will conduct the tube through the pylorus.

- Check placement of the tube in the duodenum the following day in the radiology department using X-ray with contrast.

- Documentation

Documentation in the patient record is made noting the date and signature:

- Information given to patient

- Tube position is monitored

○ Observation for aspiration is made

\section{Daily Care:}

- Information

- The patient is informed of precautions to be taken in relation to the tube.

- The patient is advised on oral care, daily care of skin and nostril as well as changing the plaster.

- Hygiene

Once a day or as required, the plaster is removed and the patient's face, nose and nostril washed and cleansed. The tube is attached to the nose and cheek so as to prevent pressure to the skin.

- Observation

Observation for signs of pressure in the nose.

Problems with tube: hiccups and nausea.

- Documentation

Documented in medical records with date and signature for:

- Above observations

- Monitoring the tube position at the $110 \mathrm{~cm}$ marking

- Information given to patient

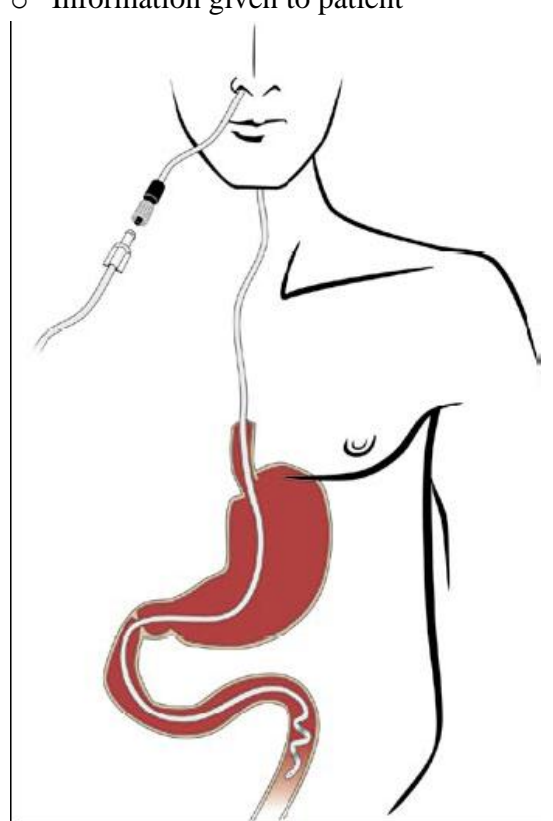

Removal of the nasogastric tube:

- The tube is removed after assessment by a doctor when the trial period is over, either because a PEG tube for continuous therapy will be inserted or therapy will be terminated.

- Information given to patient 
The patient is informed about the procedure and possible discomfort in the form of a gag reflex when the tube passes the throat and back of the tongue.

- Hygiene

The nurse and any assistants must wash and disinfect hands and use non-sterile disposable gloves for the procedure.

- Removal in the ward:

○ A tray and cellulose are supplied

- The patient must sit up or lie down and bend the head forward

- The tube is clamped

- Nasal plaster is removed

- The patient is asked to take a deep breath in

- The tube is removed in a long, steady movement

- The patient is offered something to drink afterwards.

- Nose and oral care

- The patient's nose is cleansed on the outside of any plaster residue

- The patient is offered chance to blow nose and brush teeth

- Further nasal/oral care is offered as required and according to discomfort

- Documentation

○ Documented in nursing reports with date and signature for:

- Removal of tube

- Any problems in connection with removal of the tube

- The information delivered to the patient

Responsibility and Distribution of Tasks

- Doctor's responsibility: Prescribing insertion and removal of the tube and assistance if needed.

- Nurse's responsibility: Insertion, observation, care and if applicable removal of tube. Tasks can be delegated to a nursing auxiliary/welfare and healthcare assistants according to the department's training programme and the skills of the individual.

Relevant Technical Evidence in the Field

Instructions for use “Naso-intestinal Tube" IPN-STABILIFE DUODOPA® intestinal tube for naso-intestinal placement.

\section{APPENDIX C - INSTRUCTIONS ON POST-OPERATIVE OBSERVATION AND CARE OF PATIENTS WITH PARKINSON'S DISEASE FOLLOWING INSERTION OF A PEG TUBE UNDER LOCAL ANESTHETICS FOR LCIG.}

\section{Nursing Instructions}

\section{Problem:}

The patient has had a PEG tube inserted to administer LCIG (Duodopa) medication. This does not include delivery of food and fluid.

This guideline does not include instructions concerning delivery of food and fluid in the PEG tube.

Aim:

- To ensure that, immediately after insertion of a PEG tube, the patient is correctly observed according to stipulated post-operative observational standards.

- The patients are discharged from the ward with an individually tailored plan.

Patient is given satisfactory information and patient involvement is secured.

Observations made by the staff following the transfer of the patient from the endoscopic unit to the ward, after PEG and inner tube insertion. Observations are registered in the nursing report sheet.

- Level of consciousness, colour, respiration, pulse, BP, temperature and pain.

- Insertion site is observed: colour, swelling, bleeding and secretion.

Painkillers are offered as prescribed.

NB: The external tube may not be used for medicine within the first 4 hours.

Check list for the position of the PEG tube and inner tube:

- Note in medical records which marking on the tube is at skin level. If there are no printed markings on the tube, use a waterproof felt tip pen. There must be a distance of $2-5 \mathrm{~mm}$ between the skin and the external fixation plate. For the first 14 days it is important that the tube pulled each day to the stipulated marking.

- If the tube has moved in relation to the marking: Aspirate from the external tube using a Janet syringe. (Stomach content: $\mathrm{pH}<3$. $\mathrm{pH}$ can be monitored using the same type of dipstick used to test urine). 
- If in doubt over the position of the tube, call a doctor.

\section{Skin care Around the Stoma:}

- For the first 14 days, while the channel between stomach and skin is being established, the internal and external fixation plates are kept close together (but without being too tight) so that the stomach adheres to the abdominal wall. This creates aa short channel to the skin. This prevents leakage in the abdominal cavity and skin problems due to stomach content along the tube.

- The surrounding skin is observed daily to ensure that the fixation plate is not sitting too tightly. There must be no signs of ischaemia. In some patients, onset of oedema around the insertion site is observed. This may necessitate loosening the fixation plate so that 2-5 $\mathrm{mm}$ remains between the skin and fixation plate.

- The surrounding skin must be inspected daily for signs of infection: redness, swelling, secretion. If an infection is suspected, call the doctor to assess the need for treatment.

- The fixation plate and tube are cleaned daily using soap and water (if possible, fragrance-free and $\mathrm{pH}$-neutral soap).

- Wash around the tube daily using water and, if possible, fragrance-free, $\mathrm{pH}$-neutral soap. Lift the fixation plate slightly and clean and dry the skin around the stoma using a cotton bud or gauze swab.

- Patients may shower 48 hours after insertion of tube.

- Generally, no dressing is required. A dressing may be placed over the fixation plate, never under, as this inhibits secretion from the wound and inflammation can develop around the stoma.

- Leakage of stomach content can be avoided by pulling the fixation plate close to the abdominal surface. Leakage of stomach content on the skin may also be due to gastric retention.

- After 14 days, move the tube into the channel carefully once a day in order to prevent the internal fixation plate in the ventricle becoming fixed.

\section{Handling/Moving the PEG Tube:}

- Detach the tube from the fixation plate.

- Push the tube in $2-3 \mathrm{~cm}$.

- $\quad$ Pull the PEG tube back in place close to the gastric wall.

- Attach the tube to the fixation plate. There must be a 2-5 mm distance between skin and fixation plate.

\section{Information and Instruction:}

- The patient receives instructions and is trained on how to observe and clean the PEG tube and surrounding area.

- The patient receives instructions on the administration and managing of Duodopa and how to use the pump.

- The patient receives instructions on trouble shooting regarding the pump and connectors and medication.

\section{Discharge of the Patient from the Ward with the Inserted PEG Tube and Inner Tube:}

- The patient, the patients spouse, caregiver, nursing staff, additional aid staff must be advised regarding:

- Checking position of tube and moving tube

- Administering medicine via the tube

- Where and who to contact in case of problems with blockage of the tubes, equipment breakage etc. (PEG folder)

- The patient is issued with the remaining medication and a prescription of Duodopa and Sinemet Depot.

- The patient is given the necessary equipment to take home.

\section{REFERENCES}

[1] de Lau LM, Breteler MM. Epidemiology of Parkinson's disease. Lancet Neurol 2006; 5(6): 525-35.

[2] de Rijk MC, Tzourio C, Breteler MM, et al. Prevalence of parkinsonism and Parkinson's disease in Europe: the EUROPARKINSON Collaborative Study. European Community Concerted Action on the Epidemiology of Parkinson's disease. J Neurol Neurosurg Psychiatry 1997; 62(1): 10-5.
[3] Hirtz D, Thurman DJ, Gwinn-Hardy K, Mohamed M, Chaudhuri AR, Zalutsky R. How common are the "common" neurologic disorders? Neurology 2007; 68(5): 326-37.

[4] Olanow CW, Obeso JA, Stocchi F. Continuous dopamine-receptor treatment of Parkinson's disease: scientific rationale and clinical implications. Lancet Neurol 2006; 5(8): 677-87.

[5] Nyholm D, Lennernas H, Gomes-Trolin C, Aquilonius SM. Levodopa pharmacokinetics and motor performance during activities of daily living in patients with Parkinson's disease on 
individual drug combinations. Clin Neuropharmacol 2002; 25(2): 89-96.

[6] Agerbo S, Bakke M, Bode M, et al. Parkinsons Sygdom - Klinisk vejledning. Diagnose,forløb og behandling fra et tværfagligt perspektiv. National Clinical Guideline 2011; (2.udgave).

[7] Antonini A, Isaias IU, Canesi M, et al. Duodenal levodopa infusion for advanced Parkinson's disease: 12-month treatment outcome. Mov Disord 2007; 22(8): 1145-9.

[8] Devos D. Patient profile, indications, efficacy and safety of duodenal levodopa infusion in advanced Parkinson's disease. Mov Disord 2009; 24(7): 993-1000.

[9] Fernandez HH, Odin P. Levodopa-carbidopa intestinal gel for treatment of advanced Parkinson's disease. Curr Med Res Opin 2011; 27(5): 907-19.

[10] Honig H, Antonini A, Martinez-Martin P, et al. Intrajejunal levodopa infusion in Parkinson's disease: a pilot multicenter study of effects on nonmotor symptoms and quality of life. Mov Disord 2009; 24(10): 1468-74.
[11] Lundquist C. Continuous levodopa for advanced Parkinson's disease. Neuropsychiatr Dis Treatment 2007; 3(3): 1-14.

[12] Nyholm D, Odin P. Continuous Intra-intestinal Infusion of Levodopa/Carbidopa in Advanced Parkinson's Disease. Eur Neurol Dis 2007; Parkinson's Disease: 45-8.

[13] Volkman J. The Therapeutic Place of Duodopa - Choosing the Right Therapy for Indidual Patients. Eur Neurol Rev 2008; 3(2): 29-32.

[14] Summary of Product Characteristics, Duodopa. 2010.

[15] Nyholm D. Enteral levodopa/carbidopa gel infusion for the treatment of motor fluctuations and dyskinesias in advanced Parkinson's disease. Expert Rev Neurother 2006; 6(10): 1403-11.

[16] Nyholm D, Lewander T, Johansson A, Lewitt PA, Lundqvist C, Aquilonius SM. Enteral levodopa/carbidopa infusion in advanced Parkinson disease: long-term exposure. Clin Neuropharmacol 2008; 31(2): 63-73.

Received: January 11, 2012

(C) Pedersen et al.; Licensee Bentham Open.

This is an open access article licensed under the terms of the Creative Commons Attribution Non-Commercial License (http://creativecommons.org/licenses/by-nc/3.0/) which permits unrestricted, non-commercial use, distribution and reproduction in any medium, provided the work is properly cited. 